\title{
BEM-ESTAR DE GALINHAS POEDEIRAS EM DIFERENTES DENSIDADES DE ALOJAMENTO
}

\section{BIOENG}

\author{
V.A.R. Castilho ${ }^{1}$, R.G. Garcia ${ }^{1}$, N.D.S. Lima ${ }^{2 *}$, K.C. Nunes ${ }^{3}$, F.R. \\ Caldara $^{1}$, I.A. Nääs ${ }^{1}$, B. Barreto ${ }^{1}$, F.G. Jacob ${ }^{2}$
}

${ }^{1}$ UFGD - Univ Federal da Grande Dourados, FCA, Dourados, MS, Brasil

${ }^{2}$ UNICAMP - Univ Estadual de Campinas, FEAGRI, Campinas, SP, Brasil

${ }^{3} U E M-$ Univ Estadual de Maringá, Maringá, PR, Brasil

Article history: Received 05 April 2015; Received in revised form 06 June 2015; Accepted 08 June 2015; Available online 30 June 2015.

\section{RESUMO}

O estudo foi realizado com objetivo de avaliar os parâmetros fisiológicos de poedeiras alojadas em diferentes densidades de alojamento e períodos. Foram utilizadas 150 poedeiras Dekalb White com 20 semanas de idade alojadas em um galpão convencional com gaiolas metálicas com duas subdivisões de $50 \times 45 \times 40 \mathrm{~cm}^{2}$ e distribuídas em três tratamentos: 8 aves por gaiola $\left(562,5 \mathrm{~cm}^{2} /\right.$ ave); 10 aves por gaiola $\left(450 \mathrm{~cm}^{2} /\right.$ ave) e 12 aves por gaiola (375 $\mathrm{cm}^{2} /$ ave), com cinco repetições cada. Avaliaram-se parâmetros fisiológicos (frequência respiratória, temperatura cloacal e temperatura superficial) e condições ambientais (temperatura ambiente e umidade relativa) durante três ciclos de 28 dias. Adotou-se um delineamento inteiramente casualizado com arranjo fatorial $3 \times 3$ (três densidades de alojamento e três períodos). Os resultados demonstraram que a densidade de alojamento não prejudicou a frequência respiratória e a temperatura superficial, porém, a temperatura cloacal diferiu significativamente, sendo superior às 16 horas e a densidade de alojamento com 10 aves/gaiola permaneceu intermediário às outras densidades. Os parâmetros voltados à busca do conforto térmico evidenciaram que nos horários mais quentes do dia (até as 16:00 horas), independente da densidade, as aves apresentaram temperaturas maiores que na parte da manhã.

Palavras-chave: produção de ovos, conforto térmico, condições ambientais

\section{WELFARE OF LAYING HENS IN DIFFERENT DENSITIES OF HOUSING}

\begin{abstract}
The study was carried with objective to evaluate the physiologic parameters of bird laying housed on different housing density and period. Were used 150 Dekalb White bird laying with 20 weeks of age was housed on conventional aviary with metallic cages with two subdivision of $50 \times 45 \times 40 \mathrm{~cm}^{2}$ and distribution on three treatments: 8 bird laying by cage $\left(562,5 \mathrm{~cm}^{2} /\right.$ bird); 10 bird laying by cage $\left(450 \mathrm{~cm}^{2} /\right.$ bird $)$ and 12 bird laying by cage (375 $\mathrm{cm}^{2} /$ bird), with five repetition each. Were evaluated physiologic parameters (respiratory frequency, cloacal temperature and superficial temperature) and ambience (environmental temperature and relative humidity) during three circles of 28 days. Was adopted a randomized design with factorial arrangement $3 \times 3$ (three housing density and three periods). The results demonstrate that the housing density not impaired the respiratory frequency and superficial temperature, however the cloacal temperature differed significantly, being higher at four p.m.
\end{abstract}

*nilsa.lima@feagri.unicamp.br 
and the housing density with $10 \mathrm{bird} / \mathrm{cage}$ remained intermediary with others density. The parameters aimed at achieving thermal comfort showed that at the hottest times of the day (until 16:00), regardless of the density, the birds showed higher temperatures in the morning.

Keywords: egg production, thermal comfort, environmental conditions.

\section{INTRODUÇÃO}

A redução da área de gaiola por ave, assim como da área de comedouro e bebedouro, ou seja, a alta densidade de alojamento pode causar efeito negativo no bem-estar das aves, e consequentemente na produção de ovos. Esse ambiente para a produção e o bem-estar, pode não ser compatível com as necessidades fisiológicas das aves, gerando com isto uma susceptibilidade a diferentes tipos de estresses. Entre estes agentes potenciais estressores estão os efeitos do calor, associados às altas temperaturas e umidade relativa do ar, mais conhecido como estresse térmico (FURLAN, 2005). Com o estresse térmico as galinhas reduzem o consumo e a conversão alimentar, em consequência há uma baixa taxa de crescimento, aumento no consumo de água, queda na produção e qualidade dos ovos, além de alterar as respostas fisiológicas (SILVA et al., 2005).

Aves são animais homeotérmicos, ou seja, mantém a temperatura corporal relativamente constante, independente da temperatura ambiental. A temperatura ambiental de conforto térmico para aves poedeiras encontra-se entre 21 e $28{ }^{\circ} \mathrm{C}$ (TINÔCO et. al., 2007). Nesta faixa de temperatura, os animais mantêm a produção de calor a um nível normal, o metabolismo é mais baixo. De acordo com Borges et al. (2003), a zona de termoneutralidade é influenciada por muitos fatores, incluindo a idade, ventilação, linhagem, nível nutricional, umidade e tamanho da ave.

A quantidade de energia térmica estocada por unidade da massa corporal determina a temperatura corporal da ave. Essa energia pode ser aumentada ou diminuída pelos processos de termogênese e termólise. Para manter a temperatura fisiológica, esses processos são realizados por um centro termorregulador, localizado no sistema nervoso central (ABREU \& ABREU, 2004), sendo captadas as sensações de frio e calor por células termorreceptoras periféricas (ABREU et al., 2007).

Essas trocas térmicas com o meio são realizadas por quatro componentes principais: radiação, condução e convecção, denominadas não evaporativas, e através da evaporação, que constitui um dos mais importantes mecanismos latentes de transferência térmica utilizada pelos animais (SILVA, 2008). A perda de calor latente ocorre devido a um diferencial de pressão de vapor (MAIA et al., 2005). No caso das aves, a troca por evaporação ocorre na forma da ofegação, uma vez que a ausência de glândulas sudoríparas impossibilita a sudação.

De maneira geral, as perdas de calor nas aves são controladas por meio da alteração do fluxo sanguíneo na superfície corporal, ou alterando a taxa de evaporação no trato respiratório (ABREU et al., 2007). A superfície corporal das aves é caracterizada pela presença de uma camada de penas, que têm maior importância quando as aves estão expostas a situação de frio. Portanto, a atividade vasomotora na pele coberta com penas é mínima e essas regiões podem ser caracterizadas como vasorregulatórias conservadoras (SHINDER et al., 2007). As áreas desprovidas de penas, tais como as pernas e área facial, são fundamentais no processo termorregulatório, assim como as regiões corporais altamente vascularizadas como crista e barbela.

Assim, com a troca contínua de energia com o ambiente, tornam-se fundamentais alguns ajustes fisiológicos na tentativa de manter o equilíbrio térmico (SILVA, 2008). As penas e penugem nas aves têm um papel fundamental no equilíbrio térmico entre o organismo e o 
ambiente, acarretando peculiaridades na regulação do equilíbrio térmico. Elas interferem diretamente na eficiência da radiação, da convecção e da condução como mecanismos de eliminação de calor, mas em ambientes tropicais o diferencial de temperatura entre $\mathrm{o}$ ambiente e a superfície do corpo dos animais tende a ser pequeno (MALHEIROS et al., 2000; SILVA \& SEVEGNANI, 2001) e às vezes, negativo.

A frequência respiratória também é considerada um parâmetro fisiológico importante na caracterização da condição de conforto ou estresse dos animais. As aves tendem a aumentar a frequência respiratória em até dez vezes o seu ritmo normal em resposta ao desconforto térmico, indicando o uso de mecanismos evaporativos na troca de calor (SANTOS et al., 2006). Sob elevadas temperaturas e umidade relativa, as aves podem desenvolver alcalose respiratória (aumento do $\mathrm{pH}$ do sangue) devido a alta taxa de expiração de dióxido de carbono (BORGES et al., 2003). A verificação deste parâmetro é apenas visual, o que facilita sua mensuração e consequente tomada de decisão.

Dentre as modernas tecnologias disponíveis para avaliar as condições

\section{MATERIAL E MÉTODOS}

A pesquisa foi realizada no Setor Experimental de Avicultura de Postura da Faculdade de Ciências Agrárias na Universidade Federal da Grande Dourados (FCA/UFGD), localizada em Dourados, MS. O experimento foi conduzido em galpão aberto, utilizando-se 15 gaiolas convencionais para postura (arame liso), possuindo cortinas, comedouros do tipo calha e bebedouros do tipo nipple, dispostos frontalmente e externamente à gaiola. $\mathrm{O}$ período experimental foi realizado entres os dias 17 de março e 16 de Junho de 2014.

Foram utilizadas 150 galinhas poedeiras comerciais (Gallus gallus) com 20 semanas de idade, da linhagem Dekalb White, uniformes segundo as térmicas de alojamento e sua influência sobre o bem-estar dos animais, encontra-se a termografia infravermelha (EDDY et al., 2001; N $\ddot{A} \ddot{A} S$ et al., 2014), sendo utilizada em estudos para se obter as respostas térmicas em diversas espécies (PHILLIPS \& HEATH, 2001; FERREIRA, et al., 2011).

A análise termográfica surgiu como técnica de mapeamento da temperatura superficial das aves, principalmente por ser uma forma de medição não invasiva, propiciando também a estimativa de perda de calor. A técnica tem grande importância no cálculo das transferências de calor e de massa entre as aves e o ambiente ao seu redor para o dimensionamento de sistemas de ventilação e resfriamento evaporativo, tal como a inferência sobre o manejo das aves (NASCIMENTO et al., 2011; NÄÄS et al., 2014).

Desta forma, justificam-se pesquisas que busquem melhores entendimentos das interações fisiológicas das aves no ambiente de criação. Neste sentido, essa pesquisa foi conduzida com o objetivo de avaliar as respostas fisiológicas de galinhas poedeiras leves em diferentes densidades de alojamento.

características físicas, de peso e aspectos sanitários e criadas em um único programa nutricional nas fases de cria e recria. As aves foram alojadas em gaiolas com duas subdivisões de 50 x $45 \times 40 \quad \mathrm{~cm}$, distribuídas em três grupos: 8 aves por gaiola $\left(562,5 \mathrm{~cm}^{2} / \mathrm{ave}\right) ; 10$ aves por gaiola $\left(450 \mathrm{~cm}^{2} /\right.$ ave) e 12 aves por gaiola (375 $\mathrm{cm}^{2} /$ ave). As aves passaram por um período de adaptação de 28 dias.

Todas as aves foram submetidas ao mesmo programa nutricional, formulada segundo as recomendações de ROSTAGNO et al. (2011), para poedeiras comerciais leves.

Para análise dos efeitos da densidade de alojamento sobre o bem-estar das aves poedeiras, foram avaliados os parâmetros 
frequência respiratória, temperatura cloacal, temperatura superficial, temperatura ambiente e umidade relativa do ar. Essas variáveis foram mensuradas três vezes por semana em três períodos diários (09:00h, 12:00h e 16:00h) durante todo o período experimental.

A temperatura e umidade relativa do ar foram registradas por termohigrômetro digital. A temperatura superficial foi registrada utilizando a câmera termográfica Testo®. Foram obtidas imagens termográficas cobrindo toda a extensão de uma subdivisão da gaiola. As imagens foram processadas usando o software IRSoft da Testo®, a partir de 30 pontos selecionados aleatoriamente.

A emissividade adotada foi de 0,95.

Avaliou-se a frequência respiratória das aves através da observação dos

\section{RESULTADOS E DISCUSSÃO}

Entre a primeira e quarta semanas, registraram-se temperaturas médias mais elevadas e com menor amplitude térmica. Por outro lado, durantes as próximas semanas foram encontrados menores valores, porém, com variações de até $15,7^{\circ} \mathrm{C}$ entre as médias da temperatura (Figura 1). Segundo RUSAL et al. (2011), a faixa de termoneutralidade para movimentos do peito durante 15 segundos, posteriormente multiplicado por 4 .

A temperatura cloacal foi determinada inserindo o termômetro de mercúrio a três centímetros na cloaca das aves por um período de 2 minutos. Foram escolhidas duas aves ao acaso em cada gaiola, totalizando 10 aves por tratamento.

Adotou-se um delineamento inteiramente casualizado em arranjo fatorial $3 \times 3$, sendo três tratamentos com diferentes densidades de alojamento e três períodos diários (09:00, 12:00 e 16:00 horas) com cinco repetições cada tratamento (8, 10 e 12 aves por gaiola). Os dados foram submetidos à Anova no programa computacional Assistat (versão 7.6, 2014), e para a comparação entre as médias utilizou-se o teste Tukey a $5 \%$ de probabilidade.

poedeiras situa-se entre 15 e $28^{\circ} \mathrm{C}$, o que permite inferir que no decorrer do período experimental as aves se encontraram em condição de conforto térmico. De acordo com o Manual de Manejo das Poedeiras Dekalb White, a temperatura ambiente e a umidade relativa ótima devem variar de 21 a $27^{\circ} \mathrm{C}$ e de 40 a $60 \%$, respectivamente.

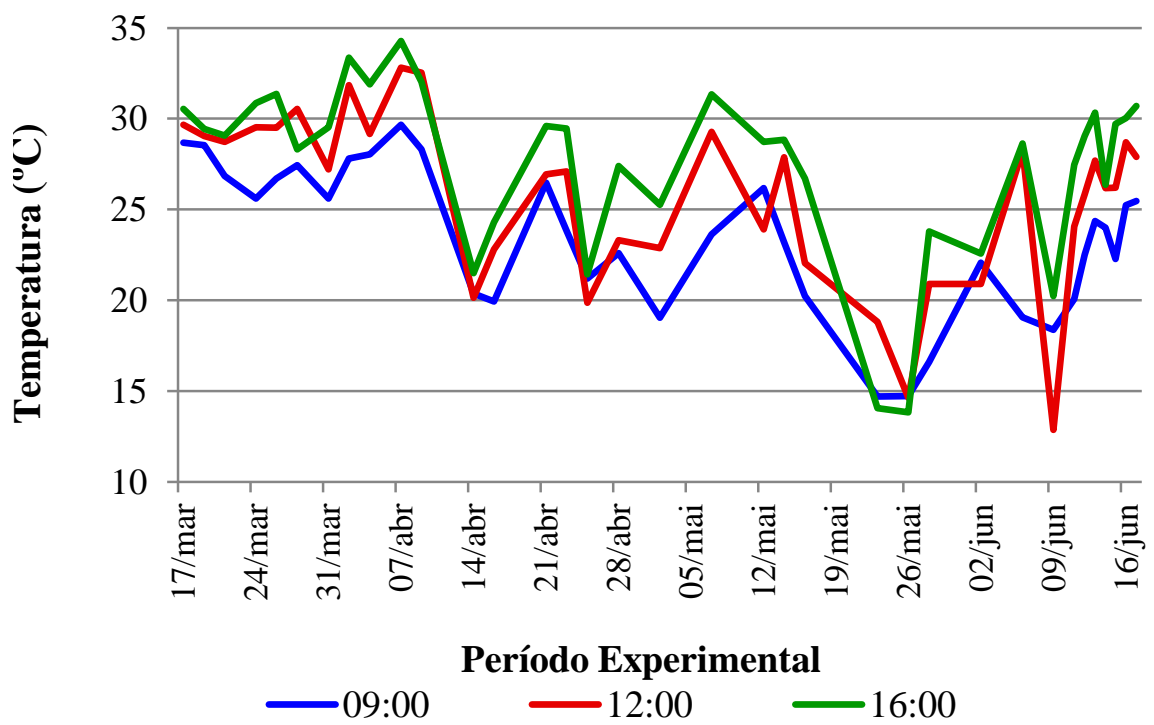

Figura 1. Médias de temperaturas $\left(\mathrm{T}^{\circ}, \mathrm{C}\right)$ durante o período de registros de dados às 09:00. 12:00 e 16:00 horas 
Ao fim da tarde observaram-se as maiores temperaturas, fato que pode ser dado pela má orientação do galpão em relação ao sol. Ao pôr do sol, a luz incide diretamente dentro do galpão, reforçando a importância da orientação leste-oeste para a construção de instalações, de modo a otimizar o aproveitamento da estrutura. Resultados de vários estudos indicam os efeitos do estresse por calor e das variações sazonais e diárias de temperatura e umidade relativa sobre o desempenho das aves (MASHALY et al., 2004; ALVES et al., 2007; CAMERINI et al., 2013).
Em relação à umidade relativa do ar, as médias observadas durante o experimento encontraram-se muito acima dos limites críticos estabelecidos na literatura e no manual dessa linhagem em praticamente todos os dias dos períodos analisados neste período. Os maiores valores de umidade relativa foram encontrados na $11^{\text {a }}$ semana, sendo notado que foi o dia mais frio registrado em todos os períodos observados, situando-se bem abaixo do limite crítico inferior dessa linhagem. Observou-se na $13^{\mathrm{a}}$ semana o aumento expressivo da umidade relativa do ar (Figura 2).

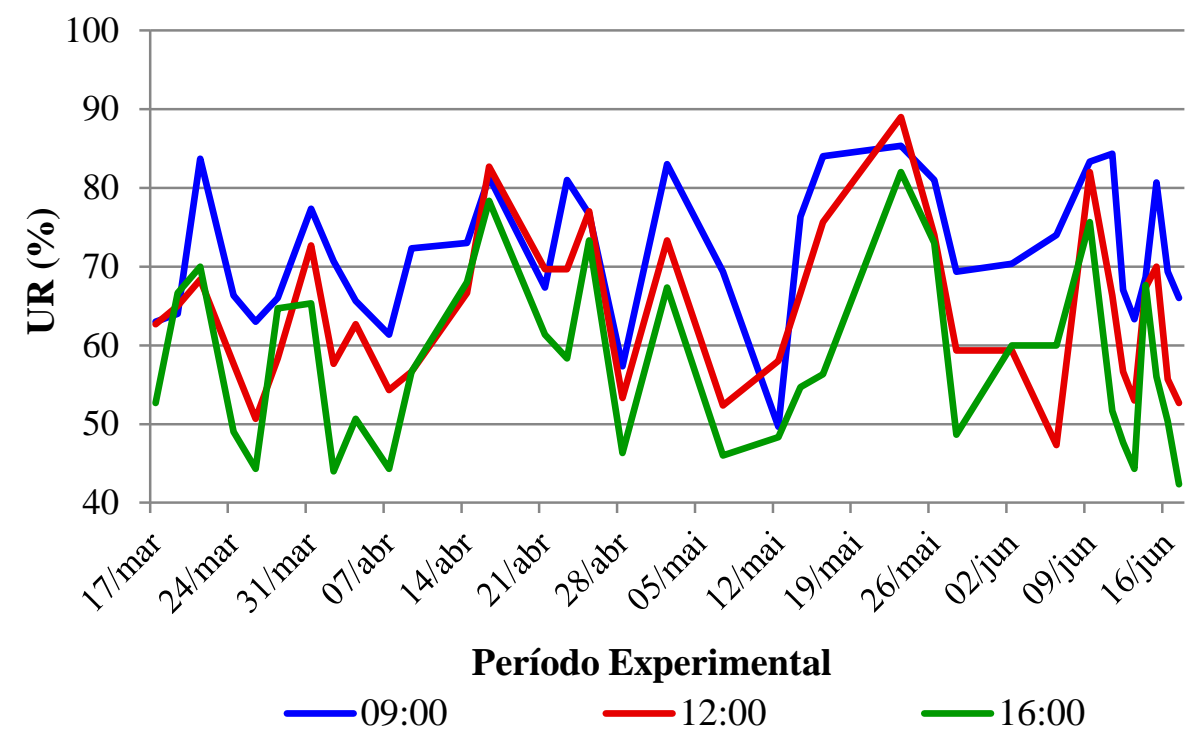

Figura 2. Médias de umidade relativa (UR \%), durante o período de registros de dados às 09:00, 12:00 e 16:00 horas

Os valores apresentados demonstram as médias das temperaturas e umidade relativa às 16 horas, havendo um pico na temperatura na $4^{\mathrm{a}}$ semana e uma queda de $12^{\circ} \mathrm{C}$ para a $5^{\mathrm{a}}$ semana, com um aumento significativo na umidade relativa. Os valores médios de umidade relativa recomendada para aves de postura da linhagem Dekalb White estão entre 40 e $65 \%$, considerando que os valores desta pesquisa estão acima do recomendado, os valores não estão dentro do conforto térmico para as galinhas em produção. Quando os valores de umidade relativa ultrapassam $80 \%$ e com oscilações constantes torna-se mais difícil para as aves manterem a homeostase e perder calor latente (JÁCOME et al., 2007; UBA, 2008).

Não houve interação entre os fatores densidade e período $(p>0,05)$. $O$ fator densidade de alojamento influenciou a temperatura cloacal, $(\mathrm{p}<0,05)$, o que está de acordo com estudos realizados por Barbosa Filho (2004). Observou-se também que não houve interação entre os fatores densidade e período $(\mathrm{p}>0,05)$.

A temperatura cloacal média observada nesse experimento foi de $41,29^{\circ} \mathrm{C}$ e temperatura mínima de $39,9^{\circ} \mathrm{C}$. De acordo com MACARI \& FURLAN (2001) o valor de $41,1^{\circ} \mathrm{C}$ de temperatura retal das aves como limite inferior da condição de estresse térmico, ou seja, 
quando este limite é ultrapassado, são desencadeados mecanismos fisiológicos para manutenção da temperatura corpórea, o que caracteriza a condição de estresse por frio.

As aves alojadas em densidade de 8 aves/gaiola apresentaram menor temperatura cloacal $\left(41,2^{\circ} \mathrm{C}\right)$ em relação aquelas alojadas em 12 aves/gaiola $\left(41,4^{\circ} \mathrm{C}\right)$, e a densidade 10 aves/gaiola mantendo-se intermediário com $41,3^{\circ} \mathrm{C}$ (Tabela 1). Todavia, o fato de ter-se mantido intermediário, indica que mesmo em temperaturas acima do recomendado, as aves conseguiram manter a homeotermia.

Tabela 1. Médias da Temperatura Cloacal (TC) das aves de acordo com as Densidades (aves/gaiolas) e Períodos do Dia (horas) avaliados durante 90 dias

\begin{tabular}{lcccc}
\hline & \multicolumn{4}{c}{ Período do Dia (horas) } \\
\cline { 2 - 5 } Densidade (aves/gaiola) & $09: 00$ & $12: 00$ & $16: 00$ & Média \\
\hline 8 aves & 41,2 & 41 & 41,3 & $41,2 \mathrm{~b}$ \\
10 aves & 41,3 & 41,1 & 41,4 & $41,3 \mathrm{ab}$ \\
12 aves & 41,2 & 41,3 & 41,4 & $41,4 \mathrm{a}$ \\
\hline Média & $41,2 \mathrm{~b}$ & $41,2 \mathrm{~b}$ & $41,4 \mathrm{a}$ & 41,29 \\
\hline CV $(\%)$ & \multicolumn{4}{c}{0,33} \\
\hline
\end{tabular}

Médias seguidas pela mesma letra na coluna não diferem estatisticamente entre si. Letras diferentes na mesma linha são estatisticamente significativas $(\mathrm{p}<0,05)$, pelo Teste de Tukey a $5 \%$ de probabilidade.

O aumento da temperatura do ar no período das 16:00 $\mathrm{h}$ afetou a temperatura cloacal diferenciando significativamente $(\mathrm{p}<0,05)$, de modo que as três densidades de alojamento apresentaram os maiores valores de temperatura (Tabela 1). Isso pode ser atribuído a orientação do galpão devido ao aumento da temperatura, e por estarem alojadas em gaiolas de arame liso (sem enriquecimento ambiental) que dificultam a troca de calor com o ambiente. Devido a isso, as tipologias dos galpões e materiais utilizados são determinantes para uma condição térmica adequada à produção da ave. $\mathrm{O}$ calor gerado pelas aves e aquele proveniente da radiação solar, são as duas fontes principais de calor no interior das instalações (TINÔCO, et al., 2007). A ave sente este calor e exprime sua resposta com baixa produtividade.

Em trabalho realizado por BATISTA et al. (2012), avaliando o comportamento de postura com a produção de avos de poedeiras em produção em dois tamanhos de grupo (6 e 12 aves) e duas densidades de alojamento (774 e $1.440 \mathrm{~cm}^{2} \mathrm{ave}^{-1}$ ), com ambiente enriquecido com ninho de caixa e poleiro, observaram que houve maior postura de ovos fora do ninho, ou seja, a interação densidade e tamanho de grupo afetou o tempo de permanência no ninho no período da tarde, sendo que grupos de 6 aves em densidade de $1.440 \mathrm{~cm}^{2} \mathrm{ave}^{-1}$ permaneceram mais tempo no ninho.

Deste modo, produção animal ideal pode ser obtida em ambiente com temperatura adequada, sem qualquer desperdício de energia metabolizável, tanto para compensar o frio como para acionar seu sistema de termorregulação a fim de resistir ao calor ambiental (NÄÄS et al, 2007; MENEZES et al., 2009).

Na Tabela 2 apresentam-se as médias da temperatura superficial das aves de acordo com as densidades (aves/gaiolas) e nos períodos do dia (horas) avaliados durante 90 dias. Não houve diferença significativa $(p>0,05)$ para a temperatura superficial com relação à densidade $(8,10$, 12 aves/gaiola), e ao período do dia, respectivamente.

Os valores de temperaturas superficiais estão de acordo com as temperaturas registradas nos períodos no decorrer do trabalho, encontrando-se dentro do limite crítico na maioria dos 
dias. Nos horários com menor temperatura ambiental (09:00h) as aves apresentam menor temperatura superficial, confirmando a relação entre a temperatura corporal e ambiental. Para SILVA (2008), a temperatura superficial depende diretamente das condições do meio, ou seja, alterações bruscas das variáveis meteorológicas no local onde o animal se encontra resultará em alterações na temperatura superficial.

Tabela 2. Médias da Temperatura Superficial (TS) das aves de acordo com as Densidades (aves/gaiolas) e períodos do dia (horas) avaliados durante 90 dias

\begin{tabular}{lcccc}
\hline \multirow{2}{*}{ Densidade (aves/gaiola) } & \multicolumn{4}{c}{ Período do Dia (horas) } \\
\cline { 2 - 5 } & $09: 00$ & $12: 00$ & $16: 00$ & Média \\
\hline 8 aves & 26,7 & 29 & 29,6 & $28,4 \mathrm{a}$ \\
10 aves & 26,7 & 29,1 & 29,7 & $28,5 \mathrm{a}$ \\
12 aves & 27,6 & 29,9 & 30,6 & $29,4 \mathrm{a}$ \\
\hline Média & $27 \mathrm{a}$ & $29,4 \mathrm{a}$ & $30 \mathrm{a}$ & 28,8 \\
\hline CV $(\%)$ & \multicolumn{5}{c}{} \\
\hline
\end{tabular}

Médias seguidas pela mesma letra na coluna não diferem estatisticamente entre si. Letras diferentes na mesma linha são estatisticamente significativas $(\mathrm{p}<0,05)$, pelo Teste de Tukey a $5 \%$ de probabilidade.

A frequência respiratória mostrou-se dentro do recomendado pela literatura. Não foi observado efeito significativo da densidade de alojamento e período do dia (p>0,05) para a frequência respiratória das aves (Tabela 3).

Segundo SILVA \& SEVEGNANI (2001), a frequência respiratória tem grande relação com o peso corporal.
Assim, uma ave de 3,4 kg apresenta uma média de 25 a 30 respirações por minuto. Estudos realizados por BARBOSA FILHO (2004) demonstraram que a frequência respiratória de poedeiras pode variar de 23 movimentos por minuto, em ambiente termoneutro $\left(20^{\circ} \mathrm{C}\right)$ a 273 movimentos por minuto, quando submetidas a temperaturas elevadas $\left(35^{\circ} \mathrm{C}\right)$.

Tabela 3. Médias da Frequência Respiratória (FR) das aves de acordo com as Densidades (aves/gaiolas) e Períodos do Dia (horas) avaliados durante 90 dias

\begin{tabular}{lcccc}
\hline \multirow{2}{*}{ Densidade (aves/gaiola) } & \multicolumn{4}{c}{ Período do Dia (horas) } \\
\cline { 2 - 5 } & $09: 00$ & $12: 00$ & $16: 00$ & Média \\
\hline 8 aves & 28,7 & 35,8 & 34,2 & 32,9 a \\
10 aves & 29,3 & 36,8 & 33,5 & 33,2 a \\
12 aves & 28,9 & 36,1 & 32,7 & $32,3 \mathrm{a}$ \\
\hline Média & $28,8 \mathrm{a}$ & $36,2 \mathrm{a}$ & $33,5 \mathrm{a}$ & 32,8 \\
\hline CV $(\%)$ & \multicolumn{4}{c}{6,2} \\
\hline
\end{tabular}

Médias seguidas pela mesma letra na coluna não diferem estatisticamente entre si. Letras diferentes na mesma linha são estatisticamente significativas $(\mathrm{p}<0,05)$, pelo Teste de Tukey a $5 \%$ de probabilidade.

Observamos que o período em que ocorreu maior taxa de movimentos por minuto, foi em que houve maior intensidade luminosa (12:00h). Porém, de acordo com dados da literatura, as frequências encontraram-se acima do recomendado, independente da densidade de alojamento das aves, para os períodos de 12:00 e 16:00 horas (SILVA \& SEVEGNANI, 2001). 
$\mathrm{O}$ aumento da frequência respiratória é uma forma de aumentar as taxas de evaporação no ar expirado e desta forma perder calor para o ambiente. Dependendo

\section{CONCLUSÃO}

O aumento da densidade de alojamento afeta negativamente a temperatura corporal das aves Dekalb White na fase de produção, dificultando a

\section{REFERÊNCIAS}

ABREU, P.G.; ABREU, V.M.N.; COLDEBELLA, A.; JAENISH, F.R.F.; PAIVA, D.P. Condições térmicas ambientais e desempenho das aves criadas em aviários com e sem o uso de forro. Arquivo Brasileiro de Medicina Veterinária e Zootecnia, v.59, p.10141020, 2007.

ABREU, P.G.; ABREU, V.M.N. Conforto Térmico para Aves. Embrapa Suínos e Aves. Comunicado Técnico 365, Dezembro, 2004.

ALBUQUERQUE, R. Tópicos importantes na produção de poedeiras comerciais. Avicultura Industrial, v.1121, n.95, 2004.

ALVES, S.P.; SILVA, I.J.O.; PIEDADE, S.M.S. Avaliação do bem-estar de aves poedeiras comerciais: efeitos do sistema de criação e do ambiente bioclimático sobre o desempenho das aves e a qualidade de ovos. Revista Brasileira Zootecnia, v.36, n.5, p.1388-1394, 2007.

BARBOSA FILHO, J.A. Avaliação do bem-estar de aves poedeiras em diferentes sistemas de produção e condições ambientais, utilizando análise de imagens. Dissertação (Mestrado). Piracicaba: ESALQ/USP, 123p. 2004.

BATISTA, E.S.; PEREIRA, D.F.; SANCHEZ, F.T.; GUIMARÃES, M.A.; NAGAI, D.K.; SOARES, N.M.; TOGASHI, C.K.; BUENO, L.G. Comportamento de uso do ninho e desempenho produtivo de poedeiras da temperatura e da umidade do ar, as aves podem aumentar sua frequência respiratória em até 10 vezes.

troca de calor com o meio, aumentando a temperatura interna das aves. Porém, não prejudicou frequência respiratória e temperatura superficial das aves.

alojadas em diferentes densidades e tamanhos de grupo. Revista Educação Agrícola Superior, v.27, n.2, p.119-123, 2012.

BORGES, S.A.; MAIORKA, A.; SILVA, A.V.F. Fisiologia do estresse calórico e a utilização de eletrólitos em frangos de corte. Ciência Rural, Santa Maria, v.33, n.5, p.975-981, 2003.

BROWN-BRANDL, T.M.; YANAGI, T.Jr.; XIN, H.; GATES, R.S.; BUCKLIN, R.A.; ROSS, G.S. A new telemetry system for measuring core body temperature in livestock and poultry. Applied Engineering in Agriculture, St. Joseph, v.19, n.5, p.583-589, 2003.

CAMERINI, N., OLIVEIRA, D., SILVA, R., NASCIMENTO, J.; FURTADO, D. Efeito do Sistema de Criação e do Ambiente Sobre a Qualidade de Ovos de Poedeiras Comerciais. Revista Engenharia na Agricultura, 21 (4), p.334-339, 2013.

CORTIZO, E.C.; BARBOSA, M.P.; SOUZA, L.A.C. Estado da arte da termografia. Fórum Patrimônio: ambiente construído e patrimônio sustentável, Belo Horizonte, v.2, n.2, p.158-193, 2008.

EDDY, A.L., VAN HOOGMOED, L.M. and SNYDER, J.R. The role of thermography in the management of equine lameness. Veterinary Journal, v.162, p.172-181. 2001. 
FERREIRA, V.M.O.S.; FRANCISCO, N.S.; BELLONI, M.; AGUIRRE, G.M.Z.; CALDARA, F.R.; NÄÄS, I.A.; GARCIA, R.G.; ALMEIDA PAZ, I.C.L.; POLYCARPO, G.V. Infrared termography applied to the evaluation of metabolic heat loss of chicksfed with different energy densities. Brazilian Journal of Poultry Science, Campinas, v.13, n.2, p.113-118, 2011.

FURLAN, R.L.; PAULILLO, A.C. II Curso de atualização em avicultura para postura comercial. São Paulo: Jaboticabal, Funep, p.96-118, 2005.

GUAHYBA, A.S. Causas e consequências do estresse na produção comercial de aves. In: IX Semana Acadêmica de Medicina Veterinária. Rio Grande do Sul, p.1-28, Nov, 2000.

MAIA, A.S.C.; SILVA, R.G.; LOUREIRO, C.M.B. Sensible and latent heat loss from the body surface of Holstein cows in a tropical environment. International Journal of Biometeorology, Heidelberg, v.50, p.17-22, 2005.

JÁCOME， I.M.T.D.; FURTADO， D.A.; LEAL, A.F.; SILVA, J.H.V.; MOURA, J.F.P. Avaliação de índices de conforto térmico de instalações para poedeiras no nordeste do Brasil. Revista Brasileira de Engenharia Agrícola e Ambiental, v.11, n.5, p.537-531, 2007.

MACARI, M.; FURLAN, R.L. Ambiência na produção de aves em clima tropical. In: SILVA, I.J. (Ed.) Ambiência na produção de aves em clima tropical. Piracicaba: FUNEP, cap. 2, p.31-87, 2001.

MALHEIROS, R.D.; MORAES, V.M.B.; BRUNO. L.D.G.; MALHEIROS, E.B.; FURLAN, R.L.; MACARI, M. Environmental temperature and cloacal and surface temperatures of broiler chicks in first week post-hatch. Journal of Applied Poultry Science, v.9, p.111-117, 2000.

MASHALY, M.M.; HENDRICKS, G.L.; KALAMA, M.A.; GEHAD, A.E.; ABBAS, A.O.; PATTERSON, P.H. Effect of heat stress on production parameters and immune responses of commercial laying hens. Poultry Science, v.83, p.889-894, 2004.

MENEZES, P.C.; CAVALCANTI, V.F.T.; LIMA, E.R.; EVENCIO NETO, J. Aspectos produtivos e econômicos de poedeiras comerciais submetidas a diferentes densidades de alojamento. Revista Brasileira de Zootecnia, Viçoca, v.38, n.11, p.2224-2229, 2009.

NÄÄS, I.A.; MIRAGLIOTTA, M.Y.; BARACHO, M. DOS S.; MOURA, D.J. Ambiência aérea em alojamento de frangos de corte: poeira e gases. Engenharia Agrícola, v.27, n.2, p.326-335, 2007.

NÄ̈̈S, I.A.; GARCIA, R.G.; CALDARA, F.R. Infrared thermal image for assessing animal health and welfare. Journal of Animal Behaviour and Biometeorology, v.2, n.3, p.66-72, 2014.

NASCIMENTO， G.R.; NÄÄS， I.A.; PEREIRA, D.F.; BARACHO, M.S.; GARCIA, R. Assessment of broilers surface temperature variation when exposed to different air temperature. Revista Brasileira de Ciência Avícola, v.13, p.259-263, 2011.

PHILLIPS, P.K.; HEATH, J.E. An infrared thermographic study of surface temperature in the euthermic woodchucks (Marmotamonax).

Comparative

Biochemistry and Pyshiology, v.129, p.557-562, 2001.

ROSTAGNO, H.S.; ALBINO, L.F.T.; DONZELE, J.L. et al. Tabelas Brasileiras para Aves e Suínos: Composição de Alimentos e Exigências Nutricionais. 3.ed. VIÇOSA: UFV, Departamento de Zootecnia, p.252, 2011.

RUSAL, M., SHINDER D., MALKA, I., YAHAV, S. Ventilation plays an important role in hens' egg production at high ambient temperature. Poultry Science, vol. 90 n.4 856-862, 2011.

SANTOS, J.R.S.; SOUZA, B.B.; SOUZA, W.H.; CEZAR, M.F.; TAVARES, G.P. Respostas fisiológicas e gradientes térmicos de ovinos das raças Santa Inês, 
Morada Nova e de seus cruzamentos com a raça Dorper às condições do semi-árido nordestino. Ciências Agrotécnicas, Lavras, v.30, n.5, p.995-1001, 2006.

SILVA, I.J.O.; SEVEGNANI, K.B. Ambiência na produção de aves de postura. In: Ambiência na produção de aves em clima tropical. Piracicaba: FUNEP, p.150-214, 2001.

SILVA, J.H.V.; JORDÃO FILHO, J.; SILVA, E.L.; RIBEIRO, M.L.G.; FURTADO, D.A. Efeito do bebedouro e da densidade de alojamento no desempenho de frangos de corte em alta temperatura. Revista Brasileira de Engenharia Agrícola e Ambiental, v.9, p.636-641. 2005.

SILVA, R.G. Biofísica ambiental - os animais e seu ambiente. Jaboticabal: Funep, 386p., 2008.

SHINDER, D.; RUSAL, M.; TANNY, J.; DRUYAN, S.; YAHAV, S. Thermoregulatory responses of chicks (Gallus domesticus) to low ambient temperatures at an early age. Poultry Science, Champaign, n.86, p.2200-2209, 2007.

TINOCO, I.F.F. Avicultura Industrial: Novos conceitos de materiais, concepções e técnicas construtivas disponíveis para galpões avícolas brasileiros. Revista Brasileira de Ciência Avícola, Campinas, v.3, n.1, p.1-26, 2007.

UNIÃO BRASILEIRA DE AVICULTURA - UBA. Protocolo de bemestar para aves poedeiras. São Paulo, 2008. Disponível em: http://www.ubabef.com.br/files/publicacoe s/e3fe1f75724db7e1483c5a1c780035f2.pdf . Acesso em: 02 junho de 2015. 